\title{
Approximating Sparsest Cut in Graphs of Bounded Treewidth
}

\author{
Eden Chlamtac* \\ Weizmann Institute
}

\author{
Robert Krauthgamer* \\ Weizmann Institute
}

\author{
Prasad Raghavendra ${ }^{\dagger}$ \\ Microsoft Research New England
}

\begin{abstract}
We give the first constant-factor approximation algorithm for Sparsest-Cut with general demands in bounded treewidth graphs. In contrast to previous algorithms, which rely on the flow-cut gap and/or metric embeddings, our approach exploits the Sherali-Adams hierarchy of linear programming relaxations.
\end{abstract}

\section{Introduction}

The Sparsest-Cut problem is one of the most famous graph optimization problems. The problem has been studied extensively due to the central role it plays in several respects. First, it represents a basic graph partitioning task that arises in several contexts, such as divide-and-conquer graph algorithms (see e.g. [LR99, Shm97] and Vaz01, Chapter 21]). Second, it is intimately related to other graph parameters, such as flows, edge-expansion, conductance, spectral gap and bisection-width. Third, there are several deep technical links between Sparsest-Cut and two seemingly unrelated concepts, the Unique Games Conjecture and Metric Embeddings.

Given that Sparsest-Cut is known to be NP-hard [MS86], the problem has been studied extensively from the perspective of polynomial-time approximation algorithms. Despite significant efforts and progress in the last two decades, we are still quite far from determining the approximability of Sparsest-Cut. This is true not only for general graphs, but also for several important graph families, such as planar graphs or bounded treewidth graphs. The latter family is the focus of this paper; we shall return to it after setting up some notation and defining the problem formally.

Problem definition. For a graph $G=(V, E)$ we let $n=|V|$. For $S \subset V$, the cutset $(S, \bar{S}) \subset V \times$ $V$ is the set of unordered pairs with exactly one endpoint in $S$, i.e. $\{\{u, v\} \in V \times V: u \in S, v \notin S\}$. In the Sparsest-Cut problem (with general demands), the input is a graph $G=(V, E)$ with edge capacities cap : $E \rightarrow \mathbb{R}_{\geq 0}$ and a set of demand pairs, $D=\left(\left\{s_{1}, t_{1}\right\}, \ldots,\left\{s_{k}, t_{k}\right\}\right)$ with a demand function dem : $D \rightarrow \mathbb{R}_{\geq 0}$. The goal is to find $S \subset V$ (a cut of $G$ ) that minimizes the ratio

$$
\Phi(S)=\frac{\sum_{(u, v) \in(S, \bar{S}) \cap E} \operatorname{cap}(u, v)}{\sum_{(u, v) \in(S, \bar{S}) \cap D} \operatorname{dem}(u, v)} .
$$

The demand function dem is often set to $\operatorname{dem}(s, t)=1$ for all $(s, t) \in D$. The special case where, in addition to this, the demand set $D$ includes all vertex pairs is referred to as uniform demands.

\footnotetext{
* This work was supported in part by The Israel Science Foundation (grant \#452/08), and by a Minerva grant. The first author was supported by a Sir Charles Clore postdoctoral fellowship. Weizmann Institute of Science, Rehovot, Israel. Email: \{eden.chlamtac, robert.krauthgamer\}@weizmann.ac.il

${ }^{\dagger}$ Email: pnagaraj@microsoft.com
} 
Treewidth. Let $G=(V, E)$ be a graph. A tree decomposition of $G=(V, E)$ is a pair $(\mathcal{B}, T)$ where $\mathcal{B}=\left\{B_{1}, \ldots, B_{m}\right\}$ is a family of subsets $B_{i} \subseteq V$ called bags, and $T$ is a tree whose nodes are the bags $B_{i}$, satisfying the following properties: (i) $V=\bigcup_{i} B_{i}$; (ii) For every edge $(u, v) \in E$, there is a bag $B_{j}$ that contains both $u, v$; and (iii) For each $v \in V$, all the bags $B_{i}$ containing $v$ form a connected subtree of $T$. The width of the tree decomposition is $\max _{i}\left|B_{i}\right|-1$. The treewidth of $G$, denoted tw $(G)$, is the smallest width among all tree decompositions of $G$. The pathwidth of $G$ is defined similarly, except that $T$ is restricted to be a path; thus, it is at least $\operatorname{tw}(G)$. It is straightforward to see that every graph $G$ excludes as a minor the complete graph on $\operatorname{tw}(G)+2$ vertices. Thus, the family of graphs of tree width $r$ contains the family of graphs with pathwidth $r$, and is contained in the family of graphs excluding $K_{r+2}$ as a minor (here $K_{r+2}$ refers to the complete graph on $r+2$ vertices).

\section{$1.1 \quad$ Results}

We present the first algorithm for general demand Sparsest-Cut that achieves a constant factor approximation for graphs of bounded treewidth $r$ (the restriction is only on the structure of the graph, not the demands). Such an algorithm is conjectured to exist by [GNRS04] (they actually make a stronger conjecture, see Section 1.3 for details). However, previously such an algorithm was not known even for $r=3$, although several algorithms are known for $r=2$ GNRS04, CJLV08, CSW10 and for bounded-pathwidth graphs [LS09 (which is a subfamily of bounded-treewidth graphs).

Theorem 1.1. There is an algorithm for Sparsest-Cut (general demands) on graphs of treewidth $r$, that runs in time $\left(2^{r} n\right)^{O(1)}$ and achieves approximation factor $C=C(r)$ (independently of $n$, the size of the graph).

Table 1 lists the best approximation algorithms known for various special cases of Sparsest-Cut. We remark that the problem (with general demands) is NP-hard even for pathwidth 2; we include a proof of this fact in Appendix A for the sake of completeness, as it is unclear whether this has appeared previously in the literature.

\begin{tabular}{|c|c|c|c|c|}
\hline Demands & Graphs & Approximation & Based on & Reference \\
\hline \multirow{6}{*}{ general } & arbitrary & $\tilde{O}(\sqrt{\log |D|})$ & SDP & ALN08 \\
\hline & treewidth 2 & 2 & LP (flow) & GNRS04, CJLV08 \\
\hline & fixed outerplanarity & $O(1)$ & LP (integer flow) & $\overline{\mathrm{CGN}^{+} 06}, \overline{\mathrm{CSW} 10}$ \\
\hline & excluding $W_{4}$-minor & $O(1)$ & LP (flow) & CJLV08 \\
\hline & fixed pathwidth & $O(1)$ & LP (flow) & LS09 \\
\hline & fixed treewidth & $O(1)$ & LP (lifted) & This work \\
\hline \multirow{4}{*}{ uniform } & arbitrary & $O(\sqrt{\log n})$ & SDP & ARV09 \\
\hline & excluding fixed-minor & $O(1)$ & LP (flow) & KPR93, FT03 \\
\hline & fixed treewidth & $\mathrm{O}(1)$ & LP (flow) & Rab03, CKS09 \\
\hline & fixed treewidth & 1 & dynamic program & ing \\
\hline
\end{tabular}

Table 1: Approximation algorithms for Sparsest-Cut. 
Techniques. Similarly to almost all previous work, our algorithm is based on rounding a linear programming (LP) relaxation of the problem. A unique feature of our algorithm is that it employs an LP relaxation derived from the hierarchy of (increasingly stronger) LPs, designed by Sherali and Adams [SA90]. Specifically, we use level $r+O(1)$ of this hierarchy. In contrast, all prior work on Sparsest-Cut uses either the standard LP (that arises as the dual of the concurrent-flow problem, see e.g. [LR99]), or its straightforward strengthening to a semidefinite program (SDP). Consequently, the entire setup changes significantly (e.g. the known connections to embeddings and flow, see Section 1.2), and we face the distinctive challenges of exploiting the complex structure of these relaxations (see Section 1.3).

While bounding the integrality gap of the standard LP (the flow-cut gap) for various graph families remains an important open problem with implications in metric embeddings (see Section 1.2), our focus is on directly approximating Sparsest-Cut. Accordingly, our LP is larger and (possibly much) stronger than the standard flow LP, and hence our rounding does not imply a bound on the flow-cut gap (akin to rounding of the SDP relaxation in ARV09, CGR08, ALN08).

Finally, note that the running time stated in Theorem 1.1 is much better than the $n^{O(r)}$ running time typically needed to solve the $r+O(1)$ level of Sherali-Adams (or any other hierarchy). The reason is that only $O\left(3^{r} n|D|\right)$ of the Sherali-Adams variables and constraints are really needed for our analysis to go through (see Remark 3.2), thus greatly improving the time needed to solve the LP. As the rounding algorithm we use is a simple variant of the standard method of randomized rounding for LP's (adapted for Sherali-Adams relaxations on bounded-treewidth graphs), the entire algorithm is both efficient and easily implementable.

\subsection{The GNRS excluded-minor conjecture}

Gupta, Newman, Rabinovich and Sinclair (GNRS) conjectured in GNRS04 that metrics supported on graphs excluding a fixed minor embed into $\ell_{1}$ with distortion $O(1)$ (i.e. independent of the graph size). By the results of [LLR95, AR98, GNRS04], this conjecture is equivalent to saying that in all such graphs (regardless of the capacities and demands), the ratio between the sparsest-cut and the concurrent-flow, called the flow-cut gap, is bounded by $O(1)$. Since the concurrent-flow problem is polynomial-time solvable (e.g. by linear programming), the conjecture would immediately imply that Sparsest-Cut admits $O(1)$ approximation (in polynomial-time) on these graphs.

Despite extensive research, the GNRS conjecture is still open, even in the special cases of planar graphs and of graphs of treewidth 3 . The list of special cases that have been resolved includes graphs of treewidth 2, $O(1)$-outerplanar graphs, graphs excluding a 4-wheel minor, and bounded-pathwidth graphs; see Table 1, where the flow LP is mentioned.

Our approximation algorithm may be interpreted as evidence supporting the GNRS conjecture (for graphs of bounded treewidth), since by the foregoing discussion, the conjecture being true would imply the existence of such approximation algorithms, and moreover that our LP's integrality gap is bounded. In fact, one consequence of our algorithm and its analysis can be directly phrased in the language of metric embeddings:

Corollary 1.2. For every $r$ there is some constant $C=C(r)$ such that every shortest-path metric on a graph of treewidth $\leq r$, for which every set of size $r+3$ is isometrically embeddable into $L_{1}$ in a locally consistent way (i.e. the embeddings of two such sets, when viewed as probability distributions over cuts, are consistent on the intersection of the sets), can be embedded into $L_{1}$ with distortion at most $C$. 
If, on the other hand, the GNRS conjecture is false, then our algorithm (and its stronger LP) gives a substantial improvement over techniques using the flow LP, and may have surprising implications for the Sherali-Adams hierarchy (see Section 1.3). Either way, our result opens up several interesting questions, which we discuss in Section 1.4.

\subsection{Related work}

Relaxation hierarchies and approximation algorithms. A research plan that has attracted a lot of attention in recent years is the use of lift-and-project methods to design improved approximation algorithms for NP-hard optimization problems. These methods, such as Sherali-Adams [SA90], Lovász-Schrijver [LS91, and Lasserre [Las02] (see [Lau03 for a comparison), systematically generate, for a given $\{0,1\}$ program (which can capture many combinatorial optimization problems, e.g. Vertex-Cover), a sequence (aka hierarchy) of increasingly stronger relaxations. The first relaxation in this sequence is often a commonly-used LP relaxation for that combinatorial problem. After $n$ steps (which are often called rounds or levels), the sequence converges to the convex hull of the integral solutions, and the $k$-th relaxation in the sequence is a convex program (LP or SDP) that can be solved in time $n^{O(k)}$. Therefore, the first few, say $O(1)$, relaxations in the sequence offer a great promise to approximation algorithms - they could be much stronger than the commonly-used LP relaxation, yet are polynomial-time computable. This is particularly promising for problems for which there is a gap between known approximations and proven hardness of approximation (or when the hardness relies on weaker assumptions than $P \neq N P$ ).

Unfortunately, starting with the work of Arora, Bollobás, Lovász, and Tourlakis [ABLT06] on Vertex-Cover, there has been a long line of work showing that for various problems, even after a large (super-constant) number of rounds, various hierarchies do not yield smaller integrality gaps than a basic LP/SDP relaxation (see, e.g. [STT07, GMPT07, Sch08, Tul09, CMM09]). In particular, Raghavendra and Steurer [RS09] have recently shown that a superconstant number of rounds of certain SDP hierarchies does not improve the integrality gap for any constraint satisfaction problem (MAX-CSP).

In contrast, only few of the known results are positive, i.e. show that certain hierarchies give a sequence of improvements in the integrality gap in their first $O(1)$ levels — this has been shown for Vertex-Cover in planar graphs [MM09, Max-Cut in dense graphs dlVK07, Knapsack KMN09, Bie08], and Maximum Matching [MS09. There are even fewer results where the improved approximation is the state-of-the-art for the respective problem — such results include recent work on Chromatic Number [Chl07], Hypergraph Independent Set [CS08, and MaxMin Allocation BCG09].

In the context of bounded-treewidth graphs, a bounded number of rounds in the Sherali-Adams hierarchy is known to be tight (i.e. give exact solutions) for many problems that are tractable on this graph family, such as CSPs WJ04. This is only partially true for Sparsest-Cut - due to the exact same reason, we easily find in the graph a cut whose edge capacity exactly matches the corresponding expression in the LP. However, the demands are arbitrary (and in particular do not have a bounded-treewidth structure), and analyzing them requires considerably more work.

Hardness and integrality gaps for sparsest-cut. As mentioned earlier, Sparsest-Cut is known to be NP-hard [MS86], and we further show in Appendix A that it is even NP-hard on graphs of pathwidth 2. Two results [KV05, CKK ${ }^{+} 06$ ] independently proved that under Khot's unique games 
conjecture Kho02], the Sparsest-Cut problem is NP-hard to approximate within any constant factor. However, the graphs produced by the reductions in these two results have large treewidth.

The standard flow LP relaxation for Sparsest-Cut was shown in [LR99] to have integrality gap $\Omega(\log n)$ in expander graphs, even for uniform demands. Its standard strengthening to an SDP relaxation (the SDP used by the known approximation algorithms of [ARV09, ALN08]) was shown in KV05, KR09, DKSV06] to have integrality gap $\Omega(\log \log n)$, even for uniform demands. For the case of general demands, a stronger bound $(\log n)^{\Omega(1)}$ was recently shown in CKN09. Some of these results were extended in CMM09, RS09 to certain hierarchies and a nontrivial number of rounds, even for uniform demands. Again, the graphs used in these results have large treewidth.

Integrality gaps for graphs of treewidth $r$ (or excluding a fixed minor of size $r$ ) follow from the above in the obvious way of replacing $n$ with $r$ (or so), for instance, the standard flow LP has integrality gap $\Omega(\log r)$. However, no stronger gaps are known for these families; in particular, it is possible that the integrality gap approaches 1 with sufficiently many rounds (depending on $r$, but not on $n$ ).

\subsection{Discussion and further questions}

We show that for the Sparsest-Cut problem, the Sherali-Adams (SA) LP hierarchy can yield algorithms with better approximation ratio than previously known. Moreover, our analysis exhibits a strong (but rather involved) connection between the input graph's treewidth and the SA hierarchy level. Several interesting questions arise immediately:

1. Can this approach be generalized to excluded-minor graphs?

2. Can the approximation factor be improved to an absolute constant (independent of the treewidth)?

A particularly intriguing and more fundamental question is whether this hierarchy (or a related one, or for a different input family) is strictly stronger than the standard LP (or SDP) relaxation. One possibility is that our relaxation can actually yield an absolute constant factor approximation (as in Question 2). Such an approximation factor is shown in CMM09 to require at least $\Omega(\log r$ ) rounds of Sherali-Adams, and we would conclude that hierarchies yield strict improvement - higher (yet constant) levels of the Sherali-Adams hierarchy do give improved approximation factors, for an increasing sequence of graph families. We note, however, that this would require a different rounding algorithm (see Remark 4.2 and Section 7). Another possibility is that the GNRS conjecture does not hold even for bounded treewidth graphs, in which case the integrality gap of the standard LP exhibits a dependence on $n$, while, as we prove here, the stronger LP does not.

\section{Technical Overview}

Relaxations arising from the Sherali-Adams (SA) hierarchy, and lift-and-project techniques in general, are known to give LP (or SDP) solutions which satisfy the following property: for every subset of variables of bounded size (bounded by the level in the hierarchy used), the LP/SDP solution restricted to these variables is a convex combination of valid $\{0,1\}$ assignments. Such a convex combination can naturally be viewed as a distribution on local assignments. In our case, for example, in an induced subgraph on $r+1$ vertices $S$, an $(r+1)$-level relaxation gives a local distribution on assignments $f: S \rightarrow\{0,1\}$ such that for every edge $(i, j)$ within $S$, the probability 
that $f(i) \neq f(j)$ is exactly the contribution of edge $(i, j)$ to the objective function (which we also call the LP-distance of this pair). Our algorithm makes explicit use of this property, which is very useful for treewidth $r$ graphs.

Given an $(r+3)$-level Sherali-Adams relaxation, for every demand pair there is some distribution which (within every bag) matches the local distributions suggested by the LP, and also cuts/separates this demand pair (i.e. assigns different values to its endpoints) with the correct probability (the LP distance). Unfortunately, there might not be any single distribution which is consistent with all demand pairs, so instead our algorithm assigns $\{0,1\}$ values at random to the vertices of the graph $G$ in a stochastic process which matches the local distributions suggested by the LP solution (per bag), but is oblivious to the structure of the demands $D$.

Intuition. To achieve a good approximation ratio, it suffices to ensure that every demand pair is cut with probability not much smaller than the its LP distance. To achieve this, the algorithm fixes an arbitrary bag as the root, and traverses the tree decomposition one bag at a time, from the root towards the leaves, and samples the assignment to currently unassigned vertices in the current bag. This assignment is sampled in a way that ignores all previous assignments to vertices outside the current bag, but achieves the correct distribution on assignments to the current bag. Essentially, the algorithm finds locally correct distributions while maximizing the entropy of the overall distribution. Intuitively, this should only "distort" the distribution suggested by the LP (for a given demand pair) only by introducing noise, which (if the noise is truly unstructured) mixes the correct global distribution with a completely random one in which every two vertices are separated with probability $\frac{1}{2}$. In this case, the probability of separating any demand pair would decrease by at most a factor 2. Unfortunately, we are not able to translate this intuition into a formal proof (and on some level, it is not accurate - see Remark 4.2). Thus we are forced to adopt a different strategy in analyzing the performance of the rounding algorithm. Let us see one illustrative special case.

Example: Simple Paths. Consider, for concreteness, the case of a single simple path $v_{1}, v_{2}, \ldots, v_{n}$. For every edge in the path $\left(v_{i-1}, v_{i}\right)$, the LP suggests cutting it (assigning different values) with some probability $p_{i}$. Our algorithm will perform the following Markov process: pick some assignment $f\left(v_{1}\right) \in\{0,1\}$ at random according to the LP, and then, at step $i$ (for $i=2, \ldots, n$ ) look only at the assignment $f\left(v_{i-1}\right)$ and let $f\left(v_{i}\right)=1-f\left(v_{i-1}\right)$ with probability $p_{i}$, and $f\left(v_{i}\right)=f\left(v_{i-1}\right)$ otherwise. Each edge has now been cut with exactly the probability corresponding to its LP distance. However, for $\left(v_{1}, v_{n}\right)$, which could be a demand pair, the LP distance between them might be much greater than the probability $q_{n}=\operatorname{Pr}\left[f\left(v_{1}\right) \neq f\left(v_{n}\right)\right]$. Let us see that the LP distance can only be a constant factor more.

First, if the above probability satisfies $q_{n} \geq \frac{1}{3}$, then clearly we are done, as all LP distances will be at most 1 . Thus we may assume that $q_{n} \leq \frac{1}{3}$. Let us examine what happens at a single step. Suppose the algorithm has separated $v_{1}$ from $v_{i-1}$ with some probability $q_{i-1} \leq \frac{1}{3}$ (assuming that all $q_{i} \leq \frac{1}{3}$ is a somewhat stronger assumption than $q_{n} \leq \frac{1}{3}$, but a more careful analysis shows it is also valid). After the current step (flipping sides with probability $p_{i}$ ), the probability that $v_{i}$ is separated from $v_{1}$ is exactly $\left(1-q_{i-1}\right) p_{i}+q_{i-1}\left(1-p_{i}\right)$. This is an increase over the previous value $q_{i-1}$ of at least

$$
\left[\left(1-q_{i-1}\right) p_{i}+q_{i-1}\left(1-p_{i}\right)\right]-q_{i-1}=\left(1-2 q_{i-1}\right) p_{i} \geq p_{i} / 3
$$


However, the LP distance from $v_{1}$ can increase by at most $p_{i}$ (by triangle inequality). Thus, we can show inductively that we never lose more than a factor 3 .

In general, our analysis will consider paths of bags of size $r+1$. Even though we can still express the distribution on assignments chosen by the rounding algorithm as a Markov process (where the possible states at every step will be assignments to some set of at most $r$ vertices), it will be less straightforward to relate the LP values to this process. It turns out that we can get a handle on the LP distances by modeling the Markov process as a layered digraph $H$ with edges capacities representing the transitions (this is only in the analysis, or in the derandomization of our algorithm). In this case the LP distance we wish to bound becomes the value of a certain $(s, t)$-flow in $H$. We then bound the flow-value from above by finding a small cut in $H$. Constructing and bounding the capacity of such a cut in $H$ constitutes the technical core of this work.

\section{The Algorithm}

\subsection{An LP relaxation using the Sherali Adams hierarchy}

Let us start with an informal overview of the Sherali-Adams (SA) hierarchy. In an LP relaxation for a $0-1$ program, the linear variables $\left\{y_{i} \mid i \in[n]\right\}$ represent linear relaxations of integer variables $x_{i} \in\{0,1\}$. We can extend such a relaxation to include variables $\left\{y_{I}\right\}$ for larger subsets $I \subseteq[n]$ (usually, up to some bounded cardinality). These should be interpreted as representing the products $\prod_{i \in I} x_{i}$ in the intended (integer) solution. Now, for any pair of sets $I, J \subseteq[n]$, we will denote by $y_{I, J}$ the linear relaxation for the polynomial $\prod_{i \in I}\left(1-x_{i}\right) \prod_{j \in J} x_{j}$. These can be derived from the variables $y_{I}$ by the inclusion-exclusion principle. That is, we define

$$
y_{I, J}=\sum_{I^{\prime} \subseteq I}(-1)^{\left|I^{\prime}\right|} y_{I^{\prime} \cup J} .
$$

The constraints defined by the polytope $\mathbf{S A}_{t}(n)$, that is, level $t$ of the Sherali-Adams hierarchy starting from the trivial $n$-dimensional LP, are simply the inclusion-exclusion constraints:

$$
\forall I, J \subseteq[n] \text { s.t. }|I \cup J| \leq t: y_{I, J} \geq 0
$$

For every solution other than the trivial (all-zero) solution, we can define a normalized solution $\left\{\tilde{y}_{I}\right\}$ as follows:

$$
\tilde{y}_{I}=y_{I} / y_{\emptyset},
$$

and the normalized derived variables $\tilde{y}_{I, J}$ can be similarly defined.

As is well-known, in a non-trivial level $t$ Sherali-Adams solution, for every set of (at most) $t$ vertices, constraints (1) imply a distribution on $\{0,1\}$ assignments to these vertices matching the LP values:

Lemma 3.1. Let $\left\{y_{I}\right\}$ be a non-zero vector in the polytope $\boldsymbol{S A}_{t}(n)$. Then for every set $L \subseteq[n]$ of cardinality $|L| \leq t$, there is a distribution $\mu_{L}$ on assignments $f: L \rightarrow\{0,1\}$ such that for all $I, J \subseteq L$,

$$
\operatorname{Pr}_{\mu_{L}}[(\forall i \in I: f(i)=0) \wedge(\forall j \in J: f(j)=1)]=\tilde{y}_{I, J} .
$$

In a Sparsest Cut relaxation, we are interested in the event in which a pair of vertices is cut (i.e. assigned different values). This is captured by the following linear variable:

$$
y_{i \neq j}=y_{\{i\},\{j\}}+y_{\{j\},\{i\}} .
$$


We can now define our relaxation for Sparsest Cut, $\mathbf{S C}_{r}(G)$ :

$$
\begin{array}{ll}
\min & \sum_{(i, j) \in E} \operatorname{cap}(i, j) y_{i \neq j} \\
\text { s.t. } & \sum_{i, j \in D} \operatorname{dem}(i, j) y_{i \neq j}=1 \\
& \left\{y_{I}\right\} \in \mathbf{S A}_{r+3}(n) \\
& y_{I, J}=y_{J, I}
\end{array}
$$

Note that constraint (3) is simply a normalization ensuring that the objective function is really a relaxation for the ratio of the two sums. Also note that constraint (5), which ensures that the LP solution is fully symmetric, does not strengthen the LP, in the following sense: For any solution $\left\{y_{I}^{\prime}\right\}$ to the above LP without constraint (司), a new solution to the symmetric LP (with the same value in the objective function) can be achieved by taking $y_{I}=\left(y_{I}^{\prime}+y_{I, \emptyset}^{\prime}\right) / 2$ without violating any of the other constraints. In particular, for every vertex $i \in V$ this gives $\tilde{y}_{i}=1-\tilde{y}_{i}=\frac{1}{2}$. While our results hold true without imposing this constraint, we will retain it as it simplifies our analysis.

Remark 3.2. The size of this LP (and the time needed to solve it) is $n^{O(r)}$. Specifically for boundedtreewidth graphs, we could also formulate a much smaller LP, where constraint (4) would be replaced with the condition $\left\{y_{I} \mid I \subseteq B \cup\{i, j\}\right\} \in \mathbf{S A}_{r+3}(r+3)$ for every bag $B$ and demand pair $(i, j) \in D$. This would reduce the size of the LP to (and time needed to solve it) to at most poly $\left(2^{r} n\right)$, and our rounding algorithm and analysis would still hold.

\subsection{Rounding the LP}

Before we present the rounding algorithm, let us introduce some notation which will be useful in describing the algorithm. This notation will allow us to easily go back-and-forth between the LP solution and the local distributions on assignments described in Lemma 3.1. For ease of notation, whenever two functions $f_{1}, f_{2}$ have disjoint domains, we will denote by $f_{1} \cup f_{2}$ the unique function from the union of the domains which is an extension of both $f_{1}$ and $f_{2}$.

- For every set of vectors $\left\{y_{I}\right\}$ and subset $L \subseteq[n]$ as in Lemma 3.1, we will denote by $\mu_{L}^{\left\{y_{I}\right\}}$ the distribution on random assignments to $L$ guaranteed by the lemma. We will omit the superscript $\left\{y_{I}\right\}$, and simply write $\mu_{L}$, when it is clear from the context.

- Conversely, for any fixed assignment $f^{\prime}: L \rightarrow\{0,1\}$, we will write $\tilde{y}_{f^{\prime}}=\tilde{y}_{L_{0}, L_{1}}$, where $L_{b}=\left\{i \in L \mid f^{\prime}(i)=b\right\}$ for $b=0,1$. Thus, for a random assignment $f: L \rightarrow\{0,1\}$ distributed according to $\mu_{L}$, we have $\operatorname{Pr}\left[f=f^{\prime}\right]=\tilde{y}_{f^{\prime}}$.

- For any nonempty subset $L^{\prime} \subseteq L$, and a given assignment $f_{0}: L \backslash L^{\prime} \rightarrow\{0,1\}$ in the support of $\mu_{L \backslash L^{\prime}}$, we will denote by $\mu_{L^{\prime}, f_{0}}$ the distribution on random assignments $f \sim \mu_{L}$ conditioned on the partial assignment $f_{0}$. Formally, a random assignment $f^{\prime}: L^{\prime} \rightarrow\{0,1\}$, distributed according to $\mu_{L^{\prime}, f_{0}}$ satisfies $\operatorname{Pr}_{f^{\prime}}\left[f^{\prime}=f_{1}\right]=\tilde{y}_{f_{0} \cup f_{1}} / \tilde{y}_{f_{0}}$ for every choice of $f_{1}: L^{\prime} \rightarrow\{0,1\}$.

Let $G$ be an graph with treewidth $r$ for some integer $r>0$, and let $(\mathcal{B}, T)$ be the corresponding tree decomposition. Let $\left\{y_{I}\right\}$ be a vector satisfying $\mathbf{S C}_{r}(G)$. We now present the rounding algorithm: 
$\operatorname{Algorithm~SC-Round}\left(G,(\mathcal{B}, T),\left\{y_{I}\right\}\right) \quad$ [Constructs a random assignment $\left.f: V \rightarrow\{0,1\}\right]$

1. Pick an arbitrary bag $B_{0} \in \mathcal{B}$ as the root of $\mathcal{T}$, and sample $\left.f\right|_{B_{0}}$ according to $\mu_{B_{0}}$.

2. Traverse the rest of the tree $T$ in any order from the root towards the leaves. For each bag $B$ traversed, do the following:

(a) Let $B^{+}$be the set of vertices in $B$ for which $f$ is already defined, and let $B^{-}=B \backslash B^{+}$. Let $f_{0}$ be the corresponding assignment $f_{0}=\left.f\right|_{B^{+}}$.

(b) If $B^{-}$is non-empty, sample $\left.f\right|_{B^{-}}$at random according to $\mu_{B^{-}, f_{0}}$.

Let us first see that every edge $(i, j) \in E$ is cut with probability exactly $\tilde{y}_{i \neq j}$. Since every edge is contained in at least one bag, it suffices to show that within every bag $B$, the assignment $\left.f\right|_{B}$ is distributed according to $\mu_{B}$. This is shown by the following straightforward lemma.

Lemma 3.3. For every bag $B$, the assignment $\left.f\right|_{B}$ produced by algorithm $S C$-Round $\left(G,(\mathcal{B}, T),\left\{y_{I}\right\}\right)$ is distributed according to $\mu_{B}$.

Proof. We show this by induction. For $B_{0}$ this holds as the assignment $\left.f\right|_{B_{0}}$ is explicitly sampled according to this distribution.

Now, let $B$ be a new bag traversed and $B^{+}$and $B^{-}$as in Step 2a. By the definition of a tree decomposition, and since the tree traversal maintains a single connected component, $B^{+}$must be fully contained in some bag $B^{\prime}$ which has already been traversed. Thus, by the inductive hypothesis, $\left.f\right|_{B^{\prime}}$ is distributed according to $\mu_{B^{\prime}}$, and in particular, $\left.f\right|_{B^{+}}$is distributed according to $\mu_{B^{+}}$. Note that this is also the marginal distribution of assignments to $B^{+}$according to $\mu_{B}$. Thus, the assignment to $\left.f\right|_{B^{+}}$must lie in the support of $\mu_{B^{+}}$(this shows that Step 2b is well defined), and for every such fixed assignment $f_{0}$, and every fixed assignment $f_{1}: B^{-} \rightarrow\{0,1\}$, we have

$$
\begin{aligned}
\operatorname{Pr}\left[\left.f\right|_{B}=f_{0} \cup f_{1}\right] & =\operatorname{Pr}\left[\left.f\right|_{B^{+}}=f_{0}\right] \cdot \operatorname{Pr}\left[\left.f\right|_{B^{-}}=f_{1}|f|_{B^{+}}=f_{0}\right] \\
& =\operatorname{Pr}_{f^{\prime} \sim \mu_{B}}\left[\left.f^{\prime}\right|_{B^{+}}=f_{0}\right] \cdot \operatorname{Pr}_{f^{\prime} \sim \mu_{B^{-}, f_{0}}}\left[\left.f^{\prime}\right|_{B^{-}}=f_{1}\right] \\
& =\operatorname{Pr}_{f^{\prime} \sim \mu_{B}}\left[\left.f^{\prime}\right|_{B^{+}}=f_{0}\right] \cdot \operatorname{Pr}_{f^{\prime} \sim \mu_{B}}\left[\left.f^{\prime}\right|_{B^{-}}=f_{1}\left|f^{\prime}\right|_{B^{+}}=f_{0}\right] \\
& =\operatorname{Pr}_{f^{\prime} \sim \mu_{B}}\left[\left.f^{\prime}\right|_{B}=f_{0} \cup f_{1}\right] .
\end{aligned}
$$

This lemma shows that the expected value of the cut is $\sum_{(i, j) \in E} \operatorname{cap}(i, j) \tilde{y}_{i \neq j}$, which is exactly the value of the objective function (2) scaled by $1 / y_{\emptyset}$. In particular, for a host of other problems where the objective function and constraints depend only on the edges (e.g. Minimum Vertex Cover, Chromatic Number), this type of LP relaxation (normalized by setting $y_{\emptyset}=1$ ), along with the above rounding, always produces an optimal solution for bounded-treewidth graphs. Thus, in some sense, we consider this to be a "natural" rounding algorithm.

Before we analyze the expected value of the cut demands (or specifically, the probability that each demand is cut), let us show that the order in which the tree $T$ is traversed has no effect on the distribution of cuts produced (it will suffice to show a slightly weaker claim - that the joint distribution of cuts in any two bags is not affected). This is shown in the following lemma.

Lemma 3.4. Let $B_{1}, B_{2} \in \mathcal{B}$ be two arbitrary bags. Then the distribution on assignments $\left.f\right|_{B_{1} \cup B_{2}}$ is invariant under any connected traversal of $T$. 
Proof. Since the set of traversed bags is always a connected component, it suffices to consider only the tree-path connecting $B_{1}$ and $B_{2}$. Let us proceed by induction on the length of the path. If $B_{1}$ and $B_{2}$ are adjacent bags, then regardless of the order in which they are traversed, for any fixed assignment $f_{0}: B_{1} \cap B_{2} \rightarrow\{0,1\}$, if $\left.f\right|_{B_{1} \cap B_{2}}=f_{0}$, then $f_{B_{1} \backslash B_{2}}$ and $f_{B_{2} \backslash B_{1}}$ are distributed according to $\mu_{B_{1} \backslash B_{2}, f_{0}}$ and $\mu_{B_{2} \backslash B_{1}, f_{0}}$, respectively. Moreover, these two assignments are independent (after conditioning on $\left.f\right|_{B_{1} \cap B_{2}}=f_{0}$ ) regardless of the order of traversal. Thus, in either ordering, the same distribution on cuts can be achieved by first sampling $\left.f\right|_{B_{1} \cap B_{2}}$ according to $\mu_{B_{1} \cap B_{2}}$, and then sampling $f_{B_{1} \backslash B_{2}}$ and $f_{B_{2} \backslash B_{1}}$ independently according to the above distributions, where $f_{0}=\left.f\right|_{B_{1} \cap B_{2}}$.

Now suppose that bags $B_{1}$ and $B_{2}$ are not adjacent, and let $B_{1}^{\prime}$ the bad adjacent to $B_{1}$ on the path to $B_{2}$. By the inductive hypothesis, the distribution on $\left.f\right|_{B_{1}^{\prime} \cup B_{2}}$ is invariant under the order in which the path between $B_{1}^{\prime}$ and $B_{2}$ is traversed. In particular, this is true for the distribution on $\left.f\right|_{\left(B_{1} \cap B_{1}^{\prime}\right) \cup\left(B_{2} \backslash B_{1}\right)}$, call it $\mathcal{F}^{\prime}$. Thus, arguing as above, we see that any ordering results in the distribution on $f_{B_{1} \cup B_{2}}$ obtained by first sampling $\left.f\right|_{B_{1} \cap B_{1}^{\prime}}$ according to $\mu_{B_{1} \cap B_{1}^{\prime}}$, and then sampling $f_{B_{1} \backslash B_{1}^{\prime}}$ according to $\mu_{B_{1} \backslash B_{1}^{\prime},\left.f\right|_{B_{1} \cap B_{1}^{\prime}}}$ and then independently sampling $f_{B_{2} \backslash B_{1}}$ according to the distribution $\mathcal{F}^{\prime}$ conditioned on the value of $\left.f\right|_{B_{1} \cap B_{1}^{\prime}}$.

\section{Markov Flow Graphs}

In this section and the next two, we shall show the following lemma, which together with Lemma 3.3 implies Theorem 1.1 (see Remark 4.3).

Lemma 4.1. For every integer $r>0$ there exists a constant $c_{r}>0$ such that for any treewidth$r$ graph $G$ with tree decomposition $(\mathcal{B}, T)$, and vectors $\left\{y_{I}\right\}$ satisfying $S C_{r}(G)$, algorithm $S C$ $\operatorname{Round}\left(G,(\mathcal{B}, T),\left\{y_{I}\right\}\right)$ outputs a random $f: V \rightarrow\{0,1\}$ s.t. for every $i, j \in V$,

$$
\operatorname{Pr}[f(i) \neq f(j)] \geq c_{r} \tilde{y}_{i \neq j} .
$$

Remark 4.2. The constant $c_{r}$ arising in our analysis is quite small (roughly $2^{-r 2^{r}}$ ). While we believe this can be improved, we cannot eliminate the dependence on $r$, as a lower bound on the performance of our rounding algorithm (see Section 7) shows that $c_{r}$ cannot be more than $2^{-r / 2}$.

Remark 4.3. In fact, Lemmas 3.3 and 4.1 taken together show the following: Given any solution to $\mathrm{SC}_{r}(G)$ with objective function value $\alpha>0$, algorithm SC-Round produces a random assignment $f$ satisfying

$$
\mathbb{E}\left[\sum_{(i, j) \in E} \operatorname{cap}(i, j)|f(i)-f(j)|-\frac{\alpha}{c_{r}} \sum_{(i, j) \in D} \operatorname{dem}(i, j)|f(i)-f(j)|\right] \leq 0 .
$$

This means the algorithm produces a $1 / c_{r}$-approximation with positive probability, but does not immediately imply a lower bound on that probability. Fortunately, following the analysis in this section, the algorithm can be derandomized by the method of conditional expectations, since, at each step, finding the probability of separating each demand pair reduces to calculating the probability of reaching a certain state at a certain phase in some Markov process, which simply involves multiplying $O(n)$ transition matrices of size at most $2^{r} \times 2^{r}$ (in fact, these can be consolidated so that every step of the algorithm involves a total of $O(n|T|)$ small matrix multiplications for all demands combined, where $T$ is the set of vertices participating in demand pairs). 
For vertices $i, j \in V$ belonging to (at least) one common bag, Lemma 3.3 implies equality in (6) for $c_{r}=1$. For $i, j \in V$ which do not lie in the same bag, consider the path of bags $B_{1}, \ldots, B_{N}$ in tree $T$ from the (connected) component of bags containing $i$ to the component of bags containing $j$. By Lemma 3.4, we may assume that the algorithm traverses the path in order from $B_{1}$ to $B_{N}$.

To understand the event that vertices $i$ and $j$ are separated, it suffices to consider the following incomplete (but consistent) description of the stochastic process involved: Let $S_{0}=\{i\}$ and $S_{N}=$ $\{j\}$, and let $S_{l}=B_{l} \cap B_{l+1}$ for $l=1, \ldots, N-1$. The algorithm assigns $f(i)$ a value in $\{0,1\}$ uniformly at random, and then for $l=1, \ldots, N$, samples $\left.f\right|_{S_{l}}$ from the distribution $\mu_{S_{l},\left.f\right|_{S_{l-1}}}$ (we extend the definition of $\mu_{S, f^{\prime}}$ in the natural way to include the case where $S$ may intersect the domain of $f^{\prime}$ ).

This is a Markov process, and can be viewed as a Markov flow graph. That is, a layered graph, where each layer consists of nodes representing the different states (in this case, assignments to $S_{l}$ ), with exactly one unit of flow going from the first to the last layer, with all edges having flow at full capacity. Since all edges in the flow graph represent pairs of assignments within the same bag, Lemma 3.3 implies that the capacity of an edge (transition) $\left(f_{1}, f_{2}\right)$ is exactly $\tilde{y}_{f_{1} \cup f_{2}}$, and the amount of flow going through each node $f_{0}$ is $\tilde{y}_{f_{0}}$. For the sake of clarity, we will refer to the elements of $V$ as vertices and to the states in the flow graph as nodes.

We now would like to analyze the contribution of a demand pair to the LP. By constraint (5), this contribution (up to a factor $\operatorname{dem}(i, j)$ ) is $\tilde{y}_{i \neq j}=2 \tilde{y}_{\{i\},\{j\}}=2 \tilde{y}_{f^{*}}$, where $f^{*}:\{i, j\} \rightarrow\{0,1\}$ is the function assigning 0 to $i$ and 1 to $j$. Now consider a layer graph as above where each edge $\left(f_{1}, f_{2}\right)$ has flow $\tilde{y}_{f^{*} \cup f_{1} \cup f_{2}}$. To see that this is indeed a flow, note that two consecutive layers along with $i$ and $j$ only involve at most $r+3$ vertices in $G$, and so by Lemma 3.1 for any $l>0$ and function $f_{2}: S_{l} \rightarrow\{0,1\}$ the incoming flow at $f_{2}$ must be $\sum_{f_{1} \in S_{l-1}} \tilde{y}_{f^{*} \cup f_{1} \cup f_{2}}=\tilde{y}_{f^{*} \cup f_{2}}$, and so is the outgoing flow. The total flow in this graph is exactly $\tilde{y}_{f^{*}}$ (half the LP contribution $\tilde{y}_{i \neq j}$ ). Moreover, for each such edge (transition) we also have $\tilde{y}_{f^{*} \cup f_{1} \cup f_{2}} \leq \tilde{y}_{f_{1} \cup f_{2}}$. Hence, the flow with values $\left\{\tilde{y}_{f^{*} \cup f_{1} \cup f_{2}}\right\}$ is a legal flow respecting the capacities $\left\{\tilde{y}_{f_{1} \cup f_{2}}\right\}$ in the Markov flow graph which represents the rounding algorithm.

Thus it suffices to show the following:

Theorem 4.4. For every integer $k>1$, there is a constant $C=C(k)>0$ such that for any symmetric Markov flow graph $G=\left(L_{0}, \ldots, L_{N}, E\right)$ representing a Markov process $X_{0}, \ldots, X_{N}$ with sources $L_{0}=\left\{s_{0}, s_{1}\right\}$ and sinks $L_{N}=\left\{t_{0}, t_{1}\right\}$ and at most $k$ nodes per layer, the total amount of capacity-respecting flow in $G$ from $s_{0}$ to $t_{1}$ can be at most $C \cdot \operatorname{Pr}\left[X_{0}=s_{0} \wedge X_{N}=t_{1}\right]$.

Applying this theorem to the Markov flow graph described above with $k=2^{r}$ immediately implies Lemma 4.1. As usual, to bound the amount of flow in a graph from above, it suffices to find a suitable cut, which is what we will do in the following section.

\section{Bounding the Cut Size}

In this section we prove Theorem 4.4 for $k=4$ (the proof for the general case appears in Section 6). For Markov flow graph $G=\left(L_{0}, \ldots, L_{N}, E\right)$ and corresponding Markov process $X_{0}, \ldots, X_{N}$ as in the theorem, for any integers $0 \leq l_{1} \leq l_{2} \leq N$, and any vertices $u \in L_{l_{1}}, v \in L_{l_{2}}$ we will let $p(u)=\operatorname{Pr}\left[X_{l_{1}}=u\right]$ be the probability of reaching $u$, and similarly, we define $p(u, v)=\operatorname{Pr}\left[X_{l_{1}}=\right.$ 
$\left.u \wedge X_{l_{2}}=v\right]$. In particular, when $l_{2}=l_{1}+1$ and $\overrightarrow{(u, v)}$ is an edge (transition) then $p(u, v)$ is also the capacity of this edge. Note that, by the symmetry of $G$, we have $p\left(s_{0}\right)=p\left(s_{1}\right)=\frac{1}{2}$.

\subsection{A potential function for Markov flow graphs}

We will define a potential function on the layers of $G$, which will allow us to rephrase Theorem 1.1 in more convenient terms. First, for any every layer $l$ and vertex $v \in L_{l}$, let us define

$$
A(v)=\operatorname{Pr}\left[X_{0}=s_{0} \mid X_{l}=v\right]-\frac{1}{2} .
$$

This function satisfies the following stochastic property:

Lemma 5.1. For and $0<l_{1}<l_{2}$ and $v \in L_{l_{2}}$ we have

$$
A(v)=\frac{\sum_{u \in L_{l_{1}}} p(u, v) A(u)}{\sum_{u \in L_{l_{1}}} p(u, v)} .
$$

Proof. By the Markov property, we have

$$
\begin{aligned}
A(v)+\frac{1}{2}=\operatorname{Pr}\left[X_{0}=s_{0} \mid X_{l_{2}}=v\right]=p\left(s_{0}, v\right) / p(v) & =\frac{1}{p(v)} \sum_{u \in L_{l_{2}}}(p(u, v) / p(u)) p\left(s_{0}, u\right) \\
& =\frac{1}{p(v)} \sum_{u \in L_{l_{2}}} p(u, v)\left(A(u)+\frac{1}{2}\right) \\
& =\frac{\sum_{u \in L_{l_{2}}} p(u, v)\left(A(u)+\frac{1}{2}\right)}{\sum_{u \in L_{l_{2}}} p(u, v)} .
\end{aligned}
$$

Now, for every layer $l=0, \ldots, N$, let us define the following potential function:

$$
\varphi(l)=\operatorname{Var}\left[A\left(X_{l}\right)\right]=\sum_{v \in L_{l}} p(v) A(v)^{2}-\left(\sum_{v \in L_{l}} p(v) A(v)\right)^{2} .
$$

The following lemma show that this potential function is monotone decreasing in $l$, and relates the decrease directly to the transitions in the Markov process:

Lemma 5.2. For all $0<l_{1}<l_{2}$, we have $\varphi\left(l_{1}\right)-\varphi\left(l_{2}\right)=\sum_{u \in L_{l_{1}}, v \in L_{l_{2}}} p(u, v)(A(u)-A(v))^{2}$.

Proof. By Lemma 5.1, we have

$$
\sum_{v \in L_{l_{2}}} p(v) A(v)=\sum_{v \in L_{l_{2}}} \sum_{u \in L_{l_{1}}} p(u, v) A(u)=\sum_{u \in L_{l_{1}}}\left(\sum_{v \in L_{l_{2}}} p(u, v)\right) A(u)=\sum_{u \in L_{l_{1}}} p(u) A(u) .
$$


Therefore, we have

$$
\begin{aligned}
\varphi\left(l_{1}\right)-\varphi\left(l_{2}\right) & =\sum_{u \in L_{l_{1}}} p(u) A(u)^{2}-\sum_{v \in L_{l_{2}}} p(v) A(v)^{2} & \\
& =\sum_{u} \sum_{v} p(u, v) A(u)^{2}-\sum_{v} p(v) A(v)^{2} & \text { since } p(u)=\sum_{v} p(u, v) \\
& =\sum_{u} \sum_{v} p(u, v)\left(A(u)^{2}+A(v)^{2}\right)-2 \sum_{v} p(v) A(v)^{2} & \text { since } p(v)=\sum_{u} p(u, v) \\
& \left.=\sum_{v} \sum_{u} p(u, v)\left(A(u)^{2}+A(v)^{2}-2 A(u) A(v)\right)\right) . & \text { by Lemma 5.1 }
\end{aligned}
$$

Recall that we want to bound the possible flow from $s_{0}$ to $t_{1}$ by $O\left(p\left(s_{0}, t_{1}\right)\right)$. We may assume that $p\left(s_{0}, t_{1}\right)<\frac{1}{4}$ (i.e. $A\left(t_{1}\right)<0$ ), since otherwise the bound is trivial. Note that, by symmetry, we have $A\left(s_{0}\right)=-A\left(s_{1}\right)$ and $A\left(t_{0}\right)=-A\left(t_{1}\right)$. Since we have only two sources and two sinks, this implies

$$
\varphi(0)-\varphi(N)=A\left(s_{0}\right)^{2}-A\left(t_{1}\right)^{2}=\frac{1}{4}-A\left(t_{1}\right)^{2}=\frac{1}{4}-\left(2 p\left(s_{0}, t_{1}\right)-\frac{1}{2}\right)^{2} \leq 2 p\left(s_{0}, t_{1}\right) .
$$

Therefore, to prove Theorem 4.4 it suffices to show

Lemma 5.3. For every $k>0$ there is some constant $C=C(k)$ such that for any $G$ as above, with $A\left(t_{1}\right)<0$, there is a cut in $G$ separating $s_{0}$ from $t_{1}$ of capacity at most $C \cdot(\varphi(0)-\varphi(N))$.

Symmetry implies that $k$ is even, and the case of $k=2$ is fairly trivial. We will first consider the simpler case of $k=4$, while the general case is shown in Section 6 .

\subsection{Treewidth 2}

Let us start with the case of $k=4$, or $r=2$. This shows some of the main ideas in the analysis for larger $k$, while still being relatively simple. It is also an non-trivial special case, as it covers seriesparallel graphs. A more careful analysis would yield a smaller constant C (we did not optimize).

Lemma 5.4. Lemma 5.3 holds for $k=4$ and $C=100$.

Proof. We may assume that $\varphi(N) \geq \frac{49}{200}$. Otherwise, since the capacity of $s_{0}$ is $\frac{1}{2}$, the cost of simply cutting the outgoing edges of $s_{0}$ is $\frac{1}{2}=C / 200 \leq C(\varphi(0)-\varphi(N))$. Let us denote $A^{*}=$ $A\left(t_{0}\right)=\sqrt{\varphi(N)}\left(\geq \frac{7}{10 \sqrt{2}}\right)$. Let us start by cutting all edges $(u, v)$ for which $|A(u)-A(v)| \geq \frac{2}{7} A^{*}$. By Lemma 5.2, the total capacity of these edges is at most

$$
\begin{aligned}
\sum_{l=1}^{t} \sum_{\substack{u \in L_{l-1}, v_{L_{l}} \\
|A(u)-A(v)| \geq \frac{2}{7} A^{*}}} p(u, v) & \leq\left(\frac{7}{2 A^{*}}\right)^{2} \sum_{l=1}^{t} \sum_{\substack{u \in L_{l-1}, v_{L_{l}} \\
|A(u)-A(v)| \geq \frac{2}{7} A^{*}}} p(u, v)(A(u)-A(v))^{2} \\
& \leq\left(\frac{7}{2 A^{*}}\right)^{2} \sum_{l=1}^{t}(\varphi(l-1)-\varphi(l))=\left(\frac{7}{2 A^{*}}\right)^{2}(\varphi(0)-\varphi(N)) .
\end{aligned}
$$


Let us examine the rest of the graph. By symmetry, and since $\varphi$ is monotone decreasing, every layer $l$ must contain some vertex $v_{l}$ such that $A\left(v_{l}\right)>A\left(t_{0}\right)=A^{*}$, and a corresponding vertex $\tilde{v}_{l}$ with $A\left(\tilde{v}_{l}\right)=-A\left(v_{l}\right)$. Consider the inner two vertices $u_{l}, \tilde{u}_{l}$ (with $A\left(v_{l}\right) \geq A\left(u_{l}\right)=-A\left(\tilde{u}_{l}\right) \geq 0$ ). Since there are no direct edges from $v_{l-1}$ to $\tilde{v}_{l}$ (the edge would be longer than $\frac{2}{7} A^{*}$ ), the flow must travel along paths using the vertices $\left\{u_{l}, \tilde{u}_{l}\right\}_{l}$. Since we have cut long edges, the $A(\cdot)$ values of these paths must pass through the interval $\left[-\frac{1}{7} A^{*}, \frac{1}{7} A^{*}\right]$. Let $l_{1}$ be the first such interval for which there is flow from $s_{0}$ to $u_{l_{1}}$. By a similar argument, any flow from $s_{0}$ to $u_{l_{1}}$ must pass through the interval $\left[\frac{3}{7} A^{*}, \frac{5}{7} A^{*}\right]$ (before layer $l_{1}$ ). Let us take the last such layer, say $l_{0}$ (it can be checked that all flow to $u_{l_{1}}$ and $\tilde{u}_{l_{1}}$ must pass through $u_{l_{0}}$ ). To cut all flow to $u_{l_{1}}, \tilde{u}_{l_{1}}$, it suffices to remove vertex $u_{l_{0}}$, or equivalently, to cut all outgoing edges from $u_{l_{0}}$. Note that for all vertices $w \in L_{l_{1}}$ we have $\left|A(w)-A\left(u_{l_{0}}\right)\right| \geq \frac{2}{7} A^{*}$, since $A\left(u_{l_{0}}\right) \in\left[\frac{3}{7} A^{*}, \frac{5}{7} A^{*}\right], A\left(v_{l_{1}}\right)>A^{*}$, and $A\left(\tilde{v}_{l_{1}}\right) \leq A\left(\tilde{u}_{l_{1}}\right) \leq A\left(u_{l_{1}}\right) \leq \frac{1}{7} A^{*}$. Hence, by Lemma 5.2, the cost of cutting vertex $u_{l_{0}}$ is at most

$$
\begin{aligned}
p\left(u_{l_{0}}\right)=\sum_{w \in L_{l_{1}}} p\left(u_{l_{0}}, w\right) & \leq\left(\frac{7}{2 A^{*}}\right)^{2} \sum_{w \in L_{l_{1}}} p\left(u_{l_{0}}, w\right)\left(A\left(u_{l_{0}}\right)-A(w)\right)^{2} \\
& \leq\left(\frac{7}{2 A^{*}}\right)^{2}\left(\varphi\left(l_{0}\right)-\varphi\left(l_{1}\right)\right) .
\end{aligned}
$$

It is easy to see that any more flow from $s_{0}$ to $t_{1}$ must start at $v_{l_{2}}$ for some layer $l_{2} \geq l_{1}$, and so we can repeat the above argument, cutting vertices with $A(\cdot)$ value in $\left[\frac{3}{7} A^{*}, \frac{5}{7}\right]$, and paying $\left(\frac{7}{2 A^{*}}\right)^{2}\left(\varphi\left(l_{i}\right)-\varphi\left(l_{i+1}\right)\right)$ each time for non-overlapping intervals $\left[l_{0}, l_{1}\right],\left[l_{2}, l_{3}\right], \ldots,\left[l_{m}, l_{m+1}\right]$, until we have severed all flow. Combining this with the cost incurred in (7), we can bound the total capacity of edges cut by $2\left(\frac{7}{2 A^{*}}\right)^{2}(\varphi(0)-\varphi(N)) \leq 100(\varphi(0)-\varphi(N))$.

To summarize the above approach, our cutting technique follows a two phase process. First, we cut all "long" edges, which helps us isolate individual paths in the flow. Then, we isolate portions of the graph where the individual paths have a large shift in $A(\cdot)$ value (e.g. move from the interval $\left[\frac{3}{7} A^{*}, \frac{5}{7} A^{*}\right]$ to the interval $\left.\left[-\frac{1}{7} A^{*}, \frac{1}{7} A^{*}\right]\right)$, and cut such paths by removing a single vertex, charging to the difference in potential along that portion of the graph.

There are a number of technical difficulties involved in extending this argument to work for larger $k$. First, we cannot isolate specific intervals through which flow must pass in an isolated path, as these depend on the $A(\cdot)$ values of other vertices in nearby layers. Secondly, even after cutting a path at some node, we are not guaranteed that there is no other path which routes flow around the node we cut. Rather than decompose the graph into isolated paths, we cut in several (roughly $k$ ) phases using a cut-and-cluster approach. Namely, after cutting, we "cluster" together all vertices (or clusters from the previous phase) in a single layer that are close together in $A(\cdot)$ value, and ensure that the number of clusters per layer which can contain flow from $s_{0}$ to $t_{1}$ decreases after every phase.

Unfortunately, our threshold for clustering vertices increases by roughly a $k^{2}$ factor after every phase, thus ultimately incurring a loss which is exponential in $k$ (or doubly-exponential in $r$ ). We note that while this does not match our $\Omega(k)\left(=\Omega\left(2^{r}\right)\right)$ lower-bound, the lower-bound at least shows that we can not expect to get any "reasonable" dependence on $r$ (say, $O(\log r)$ ) with our rounding.

\section{Performance guarantee for general bounded treewidth}

Let us begin with a simple lemma which was implicit in the analysis of Lemma 5.4 . 
Lemma 6.1. Let $\left\{\left[l_{j}^{0}, l_{j}^{1}\right]\right\}$ be a sequence of non-overlapping intervals for integers $0 \leq l_{j}^{0}<l_{j}^{1} \leq N$ and let $W_{j} \subseteq L_{l_{j}^{0}}$ be sets of nodes in layer $L_{l_{j}^{0}}$ such that for every node $w \in W_{j}$ and every node $x \in L_{l_{j}^{1}}$ we have $|A(w)-A(x)| \geq \rho$ for some $\rho>0$. Then the cost of removing all $w \in W_{j}$ for every $j$ (i.e. cutting all outgoing edges from $w$ ) is at most $\frac{1}{\rho^{2}}(\varphi(0)-\varphi(N))$.

As we will use this lemma repeatedly, for a set of edges (resp. nodes) $T$, we will call the value $p(T) /(\varphi(0)-\varphi(N))$ the relative cost of $T$, where $p(T)$ is the total capacity of the edges (resp. nodes) in $T$. Thus our goal will be to find a cut of constant relative cost.

Let us introduce some terminology and notation:

Definition 6.2. In the context of this section, the distance between two nodes $u, v$ will always refer to the value $|A(u)-A(v)|$, which we will also call the length of $(u, v)$ when $(u, v)$ is an edge. For two non-overlapping clusters (defined below), we define the distance between them to be the minimum distance between two nodes, one in each cluster.

Definition 6.3. For any $\varepsilon>0$, an $\varepsilon$-cluster is a set of nodes belonging to a single layer, such that when ordered by their respective $A(\cdot)$-values, every two consecutive nodes are at distance at most $\varepsilon$ from each other. For any cluster $X$, we will denote $A^{+}(X)=\max _{v \in X} A(v)$ and $A^{-}(X)=$ $\min _{v \in X} A(v)$, and we will refer to the value $A^{+}(X)-A^{-}(X)$ as the width of $X$.

Note that the width of any $\varepsilon$-cluster is at most $(k-1) \varepsilon$.

Definition 6.4. In a Markov flow graph as above, with some edges already cut, we will call a cluster $X$ viable if there is any capacity-respecting flow in the remaining graph from $s_{0}$ to $t_{1}$ which goes through at least one node of $X$. For any clustering of the graph, we will define the clustered capacity to be the maximum number of viable clusters per layer, over all layers.

Let us now prove Lemma 5.3

Proof of Lemma 5.9. Let us assume that $A\left(t_{1}\right)<-\frac{1}{3}$ (recall that we've assumed $A\left(t_{1}\right) \leq 0$ ). Otherwise, simply cutting the outgoing edges of $s_{0}$ yields a cut of relative cost $\frac{1}{2} /(\varphi(0)-\varphi(N)) \leq \frac{18}{5}$.

As discussed earlier, we will proceed in $k$ phases. In each phase, we will reduce the clustered capacity of the graph. Each phase will consist of first cutting some clusters (i.e. removing all outgoing edges from the nodes in these clusters), and then increasing the size of certain other clusters (by increasing the threshold for clustering).

We begin by first cutting all edges of length at least $\varepsilon_{0}$ (for some $\varepsilon_{0}>0$ to be determined soon). By Lemma 6.1, the relative cost of this cut is at most $1 / \varepsilon_{0}^{2}$. At the end of each phase $j$, we will cluster the nodes with clustering threshold $\varepsilon_{j}=\left(12 k^{2}\right)^{j} \varepsilon_{0}$, while we will require that $k \varepsilon_{k-1} \leq \frac{1}{6}$. Thus we set $\varepsilon_{0}=1 /\left(6 k\left(12 k^{2}\right)^{k-1}\right)$. As we shall see, the relative cost of the cut at phase $j$ will be at most $1 /\left(k \varepsilon_{j-1}\right)^{2}$. Thus, the total relative cost of our cut will be at most

$$
\frac{1}{\varepsilon_{0}^{2}}+\sum_{j>0} \frac{1}{k^{2} \varepsilon_{j-1}^{2}}=\frac{1}{\varepsilon_{0}^{2}}\left(1+\sum_{j>0} \frac{1}{144^{j-1} k^{4 j-2}}\right)=O\left(\frac{1}{\varepsilon_{0}^{2}}\right)=k^{O(k)} .
$$

Before describing and analyzing the individual phases, let us note that at phase $j$ every cluster has width at most $(k-1) \varepsilon_{j-1}$. Since we have cut all edges of length at least $\varepsilon_{0}$, for two clusters 
$X_{1}, X_{2}$ in consecutive layers, there can be flow from $X_{1}$ to $X_{2}$ only if $A^{-}\left(X_{2}\right)-A^{+}\left(X_{1}\right)<\varepsilon_{0}$ and $A^{-}\left(X_{1}\right)-A^{+}\left(X_{2}\right)<\varepsilon_{0}$. In particular, when there is such flow, we have

$$
\max \left\{\left|A^{+}\left(X_{2}\right)-A^{+}\left(X_{1}\right)\right|,\left|A^{-}\left(X_{2}\right)-A^{-}\left(X_{1}\right)\right|\right\} \leq(k-1) \varepsilon_{j-1}+\varepsilon_{0} \leq k \varepsilon_{j-1} .
$$

We now proceed by induction on the clustered capacity. If the clustered capacity is 1 , then there is a single "path" of clusters from $s_{0}$ to $t_{1}$. Let $j$ be the current phase $(1 \leq j \leq k)$. By our choice of $\varepsilon_{j-1}$, and by (9), the value $A^{+}(X)$ of any $\left(\varepsilon_{j-1^{-}}\right)$cluster in the path can increase or decrease by at most $k \varepsilon_{k-1} \leq \frac{1}{6}$ at each step. Since this value starts at $A\left(s_{0}\right)=\frac{1}{2}$, and ends at $A\left(t_{1}\right)<-\frac{1}{3}$, at some point it must pass through the interval $\left[0, \frac{1}{6}\right]$. Call this layer $l_{1}$, and the corresponding cluster $X_{l_{1}}$. Now $A^{+}\left(X_{l+1}\right) \leq \frac{1}{6}$, and since the width of this cluster is at most $\frac{1}{6}$, we also have $A^{-}\left(X_{l+1}\right) \geq-\frac{1}{6}$. Thus all nodes in the cluster are at distance at least $\frac{1}{6}$ from $t_{0}$ and $t_{1}$. Thus, by Lemma 6.1, the relative cost of cutting all flow along the path by removing $X_{l_{1}}$ is at most 36 .

Now suppose the clustered capacity is $k^{\prime}$ for some $1<k^{\prime} \leq k$, and let $j$ denote the current phase. For any given layer, if the number of viable $\varepsilon_{j-1}$-clusters is strictly less than $k^{\prime}$, then we are done with that particular layer. Otherwise, if there are $k^{\prime}$ viable clusters in a layer and any two of them are at distance at most $\varepsilon_{j}$ from each other, then again we are done with that layer, since at the end of the phase the two clusters will be merged (possibly along with additional clusters) into a single $\varepsilon_{j}$-cluster. Thus, we only need to reduce the number of viable clusters in layers which contain $k^{\prime}$ distinct viable $\varepsilon_{j-1}$-clusters whose pairwise distances are all greater than $\varepsilon_{j}$.

Let us denote the first such layer by $l_{1}$, and the viable clusters by $X_{1}^{l_{1}}, \ldots, X_{k^{\prime}}^{l_{1}}$ in increasing order of $A(\cdot)$ values. Note that any viable cluster $X_{1}$ must have a corresponding viable cluster $X_{1}^{\prime}$ in the subsequent layer satisfying (9). Moreover, for any two $\varepsilon_{j-1}$-clusters $X_{1}, X_{2}$ in layer $L_{l_{1}}$ such that $A^{+}\left(X_{1}\right)<A^{-}\left(X_{2}\right)-\frac{\varepsilon_{j}}{3}$ with flow into clusters $X_{1}^{\prime}, X_{2}^{\prime}$, respectively, in the subsequent layer, there cannot be any flow from $X_{1}$ to $X_{2}^{\prime}$ or from $X_{2}$ to $X_{1}^{\prime}$. Indeed, the distance from, say, $X_{1}$ to $X_{2}^{\prime}$ is greater than $\frac{\varepsilon_{j}}{3}-k \varepsilon_{j-1}>\varepsilon_{j-1} \geq \varepsilon_{0}$. In particular, for any layer containing $k^{\prime}$ viable clusters with pairwise distance greater than $\frac{\varepsilon_{j-1}}{3}$, each cluster must have flow into exactly one cluster in the subsequent layer (there cannot be more, since no layer contains more than $k^{\prime}$ viable clusters in this phase).

Let us denote by $l_{2}$ the first layer after $l_{1}$ in which some pair of adjacent viable clusters $X_{i^{\prime}}^{l_{2}}, X_{i^{\prime}+1}^{l_{2}}$ are at distance at most $\frac{\varepsilon_{j}}{3}$. By the above argument, all viable flow in the portion of the graph from $L_{l_{1}}$ to $L_{l_{2}}$ flows through $k^{\prime}$ disjoint cluster-paths $X_{i}^{l_{1}} \rightarrow X_{i}^{l_{1}+1} \rightarrow \ldots \rightarrow X_{i}^{l_{2}}\left(\right.$ for $\left.i=1, \ldots, k^{\prime}\right)$. Therefore, to reduce the number of viable clusters in all layers $L_{l_{1}}, \ldots, L_{l_{2}}$, it suffices to cut just one cluster $X_{i}^{l}$ for some $i \in\left\{1, \ldots, k^{\prime}\right\}$ and some $l_{1}<l<l_{2}$. Consider the pair of clusters $X_{i^{\prime}}^{l_{2}}, X_{i^{\prime}+1}^{l_{2}}$. Since these clusters are at distance at most $\frac{\varepsilon_{j}}{3}$ and the corresponding clusters $X_{i^{\prime}}^{l_{1}}, X_{i^{\prime}+1}^{l_{1}}$ are at distance at least $\varepsilon_{j}$, either $A^{+}\left(X_{i^{\prime}}^{l_{2}}\right)-A^{+}\left(X_{i^{\prime}}^{l_{1}}\right) \geq \frac{\varepsilon_{j}}{3}$, or $A^{-}\left(X_{i^{\prime}+1}^{l_{1}}\right)-A^{-}\left(X_{i^{\prime}+1}^{l_{2}}\right) \geq \frac{\varepsilon_{j}}{3}$. Without loss of generality, suppose the former.

Now consider the open real interval $\left(A^{+}\left(X_{i^{\prime}}^{l_{1}}\right), A^{+}\left(X_{i^{\prime}}^{l_{2}}\right)\right)$. It has length at least $\frac{\varepsilon_{j}}{3}$, and contains at most $k-1$ values in $\left\{A(v) \mid v \in L_{l_{2}}\right\}$ (at least one node $v$ in layer $L_{l_{2}}$ has $A(v)=A^{+}\left(X_{i^{\prime}}^{l_{2}}\right)$ ). Therefore there is an open subinterval $\left(a_{0}, a_{1}\right)$ of length at least $\frac{\varepsilon_{j}}{3 k}=4 k \varepsilon_{j-1}$ containing none of these values. By (9), there must be some layer $L_{l}$ (for some $l_{1}<l<l_{2}$ ) for which $A^{+}\left(X_{i^{\prime}}^{l}\right) \in$ $\left(a_{0}+2 k \varepsilon_{j-1}, a_{0}+3 k \varepsilon_{j-1}\right)$. Since $X_{i^{\prime}}^{l}$ has width at most $k \varepsilon_{j-1}$, it must also satisfy $A^{-}\left(X_{i^{\prime}}^{l}\right) \in$ $\left(a_{0}+k \varepsilon_{j-1}, a_{0}+3 k \varepsilon_{j-1}\right)$. In particular, all nodes in $X_{i^{\prime}}^{l}$ are at distance at least $k \varepsilon_{j-1}$ from all nodes in layer $L_{l_{2}}$. We can now repeat this argument for layers $>l_{2}$, until we have exhausted all layers in the graph, and by Lemma 6.1, the relative cost of the cut will be at most $1 /\left(k \varepsilon_{j-1}\right)^{2}$, as required. 


\section{A Lower Bound for the Rounding Algorithm}

In this section, we give a lower bound on the quality of approximation of algorithm SC-Round. It is not known whether this can be translated into an integrality gap for our LP (in fact, for our construction, the integrality gap is 1). We start by showing that the reduction to Markov flows discussed in Section 4 goes both ways. Specifically, we show the following lemma:

Lemma 7.1. Let $r>0$ be a positive integer, let $H=\left(L_{0}, \ldots, L_{N}, E\right)$ be a symmetric Markov flow graph with sources $L_{0}=\left\{s_{0}, s_{1}\right\}$ and sinks $L_{N}=\left\{t_{0}, t_{1}\right\}$ with at most $2^{r}$ states per layer, and let $F$ be a capacity-respecting flow from $s_{0}$ to $t_{1}$. Then there is a graph $G$ of pathwidth at most $2 r-1$ with one demand pair $(s, t)$ and a feasible (but not necessarily optimal) solution $\left\{y_{I}\right\}$ to $\boldsymbol{S C}_{2 r}(G)$ such that $H$ represents the distribution on assignments found by algorithm SC-Round for $G$ and $\left\{y_{I}\right\}$, and $\tilde{y}_{s \neq t} \geq|F|$ (the amount of flow in $F$ ).

Proof. Let $F_{\text {sym }}=\frac{1}{2}(F+\bar{F})$ (where $\bar{F}$ is the flow from $s_{1}$ to $t_{0}$ corresponding symmetrically to $F$ ). By the symmetry of $H$, this is also a capacity-respecting (multi-)flow. Since the capacities in $H$ are themselves a flow, the residual capacity in $H$ can be decomposed into two multiflows $F_{\neq}$and $F_{=}$(between opposite and same-side terminals, respectively). As before, we may assume that both multiflows are symmetric. Thus, $H$ can be decomposed into a sum of flows $F_{\text {sym }}+F_{\neq}+F_{=}$from $\left\{s_{0}, s_{1}\right\}$ to $\left\{t_{0}, t_{1}\right\}$ where the total amount of flow between opposite terminals is $\left|F_{\text {sym }}\right|+\left|F_{\neq}\right| \geq$ $\left|F_{\text {sym }}\right|=|F|$.

Now define a graph $G$ on vertices $\bigcup_{i=0}^{N} B_{i}^{\prime}$, where $B_{0}^{\prime}=\{s\}, B_{N}^{\prime}=\{t\}$, and for all $0<i<N$, $\left|B_{i}^{\prime}\right|=r$, and some edge set which admits a path-decomposition with bags $B_{i}=B_{i}^{\prime} \cup B_{i+1}^{\prime}$. Then for an appropriate symmetry-preserving correspondence between nodes of $H$ and local assignments to vertices of $G$, every path from $\left\{s_{0}, s_{1}\right\}$ to $\left\{t_{0}, t_{1}\right\}$ in $H$ corresponds to a full assignment $f: \bigcup B_{i}^{\prime} \rightarrow$ $\{0,1\}$. Thus the flow decomposition above can be viewed as a symmetric distribution on paths, which corresponds to a symmetric distribution on $\{0,1\}$ assignments in $G$. Let $\left\{\tilde{y}_{I}\right\}$ be the (level $n$ ) Sherali-Adams solution corresponding to this distribution. Note that $\tilde{y}_{s \neq t}=\left|F_{\text {sym }}\right|+\left|F_{\neq}\right| \geq|F|$. It is also not hard to see that algorithm SC-Round given the path decomposition and any scaling of $\left\{\tilde{y}_{I}\right\}$ will produce a distribution on assignments corresponding to the flow graph $H$. Thus, letting $\left\{y_{I}\right\}$ be an appropriate scaling (satisfying constraint (3)) completes the proof.

By the above lemma, to get a lower-bound for our rounding which is exponential in the treewidth of the graph, it suffices, for every even integer $k \geq 4$, to construct a Markov flow graph as in Theorem 4.4 with at most $k$ nodes per layer, which admits $\Omega(k)\left(\frac{1}{2}+A\left(t_{1}\right)\right)$ units of capacityrespecting flow from $s_{0}$ to $t_{1}\left(\left(\frac{1}{2}+A\left(t_{1}\right)\right)\right.$ is the probability that the Markov chain starts and ends at opposite terminals $\left(s_{0}, t_{1}\right)$ or $\left(s_{1}, t_{0}\right)$ - see Section ?? for the definition of $\left.A(\cdot)\right)$. Let us see such a construction now.

Construction For every sufficiently large integer $N$ and $\varepsilon \in\left(0, \frac{1}{2(N+k)}\right)$, let $H_{k}(N, \varepsilon)$ be the following layered capacitated digraph: As before, the nodes will consist of layers $L_{0}, L_{1}, \ldots, L_{N}$ where $L_{0}=\left\{s_{0}, s_{1}\right\}$ and $L_{N}=\left\{t_{0}, t_{1}\right\}$. For every $0<j<N$, we have $L_{l}=\left\{v_{0}^{j}, v_{1}^{j}, \ldots, v_{k-1}^{j}\right\}$.

For every $i=1, \ldots, k-2$ we add (directed) edges $\left(s_{0}, v_{i}^{1}\right)$ and $\left(s_{1}, v_{k-1-i}^{1}\right)$ with capacities $2 \varepsilon(k-1-i) /(k-1)$ (respectively). We also add edges $\left(s_{0}, v_{0}^{1}\right)$ and $\left(s_{1}, v_{k-1}^{1}\right)$ each with capacity $\frac{1}{2}-(k-2) \varepsilon$. Next, for between every two consecutive layers $L_{j}, L_{j+1}($ for $1 \leq j \leq N-2$ ) we add the following directed edges: 
- For all $i=1, \ldots, k-2$ add edges $\left(v_{i-1}^{j}, v_{i}^{j+1}\right)$ and $\left(v_{i+1}^{j}, v_{i}^{j+1}\right)$ each with capacity $\varepsilon$.

- Add edges $\left(v_{0}^{j}, v_{0}^{j+1}\right)$ and $\left(v_{k-1}^{j}, v_{k-1}^{j+1}\right)$ each with capacity $\frac{1}{2}-(j+k-2) \varepsilon$.

- Add edges $\left(v_{1}^{j}, v_{1}^{j+1}\right)$ and $\left(v_{k-2}^{j}, v_{k-2}^{j+1}\right)$ each with capacity $j \varepsilon$.

Finally, for $i=0, \ldots, \frac{k}{2}-1$ add edges $\left(v_{i}^{N-1}, t_{0}\right)$ and $\left(v_{k-1-i}^{N-1}, t_{1}\right)$ with the full capacity of the respective layer $L_{N-1}$ node (i.e. capacity $2 \varepsilon$ for $i=2, \ldots, k-3$; capacity $\frac{1}{2}-(N+k-4) \varepsilon$ for $i=0, k-1$; and capacity $N \varepsilon$ for $i=1, k-2)$.

From the above construction, the definition of $A(\cdot)$, and Lemma 5.1, the following claim follows immediately:

Claim 7.2. In flow graph $H_{k}(N, \varepsilon)$ we have

1. for all layers $j=1, \ldots N-1$ and all $i=0, \ldots, k$ we have $A\left(v_{i}^{j}\right)=\frac{1}{2}-\frac{i}{k-1}$, and

2. $A\left(t_{0}\right)=\frac{1}{2}-\left(N+\left(\frac{k}{2}-2\right)\left(\frac{k}{2}+2\right)\right) \varepsilon / k$ and $A\left(t_{1}\right)=-\frac{1}{2}+\left(N+\left(\frac{k}{2}-2\right)\left(\frac{k}{2}+2\right)\right) \varepsilon /(k-1)$.

Thus, if we take $N=\omega\left(k^{2}\right)$ then

$$
\frac{1}{2}+A\left(t_{1}\right)=(1+o(1)) N \varepsilon /(k-1) .
$$

On the other hand, consider the flow (written as a weighted sum of paths) $F=\varepsilon \sum_{j=1}^{N-k} p_{j}$, where path $p_{j}$ is defined as

$$
p_{j}=s_{0} \rightarrow v_{0}^{1} \rightarrow \ldots \rightarrow v_{0}^{j} \rightarrow v_{1}^{j+1} \rightarrow v_{2}^{j+2} \rightarrow \ldots \rightarrow v_{k-2}^{j+k-2} \rightarrow v_{k-2}^{j+k-1} \rightarrow \ldots \rightarrow v_{k-2}^{N-1} \rightarrow t_{1} .
$$

It can readily be checked that $F$ is a capacity-respecting flow in $H_{k}(N, \varepsilon)$ and that $|F|=(N-k) \varepsilon$. Thus, by (10), for $N=\omega\left(k^{2}\right)$ we have $|F| \geq(1-o(1))(k-1)\left(\frac{1}{2}+A\left(t_{1}\right)\right)$, which is what we wanted to show.

Acknowledgments We would like to thank Claire Mathieu for a series of helpful conversations.

\section{References}

[ABLT06] S. Arora, B. Bollobás, L. Lovász, and I. Tourlakis. Proving integrality gaps without knowing the linear program. Theory of Computing, 2(1):19-51, 2006.

[ALN08] S. Arora, J. R. Lee, and A. Naor. Euclidean distortion and the sparsest cut. J. Amer. Math. Soc., 21(1):1-21, 2008.

[AR98] Y. Aumann and Y. Rabani. An $O(\log k)$ approximate min-cut max-flow theorem and approximation algorithm. SIAM J. Comput., 27(1):291-301, 1998.

[ARV09] S. Arora, S. Rao, and U. Vazirani. Expander flows, geometric embeddings and graph partitioning. J. $A C M, 56(2): 1-37,2009$.

[BCG09] M. Bateni, M. Charikar, and V. Guruswami. Maxmin allocation via degree lower-bounded arborescences. In 41st annual ACM symposium on Theory of computing, pages 543-552, New York, NY, USA, 2009. ACM.

[Bie08] D. Bienstock. Approximate formulations for 0-1 knapsack sets. Oper. Res. Lett., 36(3):317-320, 2008. 
$\left[\mathrm{CGN}^{+} 06\right]$ C. Chekuri, A. Gupta, I. Newman, Y. Rabinovich, and A. Sinclair. Embedding k-outerplanar graphs into $\ell_{1}$. SIAM J. Discret. Math., 20(1):119-136, 2006.

[CGR08] S. Chawla, A. Gupta, and H. Räcke. Embeddings of negative-type metrics and an improved approximation to generalized sparsest cut. ACM Transactions on Algorithms, 4(2), 2008.

[Chl07] E. Chlamtac. Approximation algorithms using hierarchies of semidefinite programming relaxations. In 48th Annual IEEE Symposium on Foundations of Computer Science, pages 691-701, 2007.

[CJLV08] A. Chakrabarti, A. Jaffe, J. R. Lee, and J. Vincent. Embeddings of topological graphs: Lossy invariants, linearization, and 2-sums. In 49th Annual IEEE Symposium on Foundations of Computer Science, pages 761-770, 2008.

$\left[\mathrm{CKK}^{+} 06\right]$ S. Chawla, R. Krauthgamer, R. Kumar, Y. Rabani, and D. Sivakumar. On the hardness of approximating multicut and sparsest-cut. Computational Complexity, 15(2):94-114, 2006.

[CKN09] J. Cheeger, B. Kleiner, and A. Naor. A $(\log n)^{\Omega(1)}$ integrality gap for the sparsest cut SDP. In 50th Annual IEEE Symposium on Foundations of Computer Science, volume 0, pages 555-564. IEEE, 2009.

[CKS09] C. Chekuri, S. Khanna, and F. B. Shepherd. A note on multiflows and treewidth. Algorithmica, 54(3):400-412, 2009.

[CMM09] M. Charikar, K. Makarychev, and Y. Makarychev. Integrality gaps for Sherali-Adams relaxations. In 41st ACM symposium on Theory of computing, pages 283-292, 2009.

[CS08] E. Chlamtac and G. Singh. Improved approximation guarantees through higher levels of SDP hierarchies. In 11th International Workshop on Approximation Algorithms for Combinatorial Optimization Problems (APPROX), pages 49-62, 2008.

[CSW10] C. Chekuri, B. Shepherd, and C. Weibel. Flow-cut gaps for integer and fractional multiflows. In 21st ACM-SIAM Symposium on Discrete Algorithms, 2010.

[DKSV06] N. R. Devanur, S. A. Khot, R. Saket, and N. K. Vishnoi. Integrality gaps for sparsest cut and minimum linear arrangement problems. In 38th Annual ACM Symposium on Theory of Computing, pages 537-546, 2006.

[dlVK07] W. F. de la Vega and C. Kenyon-Mathieu. Linear programming relaxations of maxcut. In 18th ACM-SIAM symposium on Discrete algorithms, pages 53-61, 2007.

[FT03] J. Fakcharoenphol and K. Talwar. Improved decompositions of graphs with forbidden minors. In 6th International workshop on Approximation algorithms for combinatorial optimization, pages 36-46, 2003.

[GMPT07] K. Georgiou, A. Magen, T. Pitassi, and I. Tourlakis. Integrality gaps of 2 - o(1) for vertex cover SDPs in the Lovász-Schrijver hierarchy. In 48th Annual IEEE Symposium on Foundations of Computer Science, pages 702-712, 2007.

[GNRS04] A. Gupta, I. Newman, Y. Rabinovich, and A. Sinclair. Cuts, trees and $\ell_{1}$-embeddings of graphs. Combinatorica, 24(2):233-269, 2004.

[Kho02] S. Khot. On the power of unique 2-prover 1-round games. In 34th Annual ACM Symposium on the Theory of Computing, pages 767-775, July 2002.

[KMN09] A. R. Karlin, C. Mathieu, and C. T. Nguyen. Integrality gaps of linear and semi-definite programming relaxations for knapsack. Manuscript, 2009.

[KPR93] P. Klein, S. A. Plotkin, and S. Rao. Excluded minors, network decomposition, and multicommodity flow. In 25th Annual ACM Symposium on Theory of Computing, pages 682-690, May 1993. 
[KR09] R. Krauthgamer and Y. Rabani. Improved lower bounds for embeddings into $L_{1}$. SIAM J. Comput., 38(6):2487-2498, 2009.

[KV05] S. Khot and N. K. Vishnoi. The unique games conjecture, integrality gap for cut problems and the embeddability of negative type metrics into $\ell_{1}$. In 46 th IEEE Annual Symposium on Foundations of Computer Science, pages 53-62, 2005.

[Las02] J. B. Lasserre. Semidefinite programming vs. LP relaxations for polynomial programming. Math. Oper. Res., 27(2):347-360, 2002.

[Lau03] M. Laurent. A comparison of the Sherali-Adams, Lovász-Schrijver, and Lasserre relaxations for 0-1 programming. Math. Oper. Res., 28(3):470-496, 2003.

[LLR95] N. Linial, E. London, and Y. Rabinovich. The geometry of graphs and some of its algorithmic applications. Combinatorica, 15(2):215-245, 1995.

[LR99] T. Leighton and S. Rao. Multicommodity max-flow min-cut theorems and their use in designing approximation algorithms. J. ACM, 46(6):787-832, 1999.

[LS91] L. Lovász and A. Schrijver. Cones of matrices and set-functions and 0-1 optimization. SIAM J. Optim., 1(2):166-190, 1991.

[LS09] J. R. Lee and A. Sidiropoulos. Pathwidth, trees, and random embeddings. CoRR, abs/0910.1409, 2009.

[MM09] A. Magen and M. Moharrami. Robust algorithms for maximum independent set on minor-free graphs based on the Sherali-Adams hierarchy. In 12th International Workshop on Approximation Algorithms for Combinatorial Optimization Problems (APPROX), pages 258-271, 2009.

[MS86] V. D. Milman and G. Schechtman. Asymptotic theory of finite-dimensional normed spaces. Springer-Verlag, Berlin, 1986.

[MS09] C. Mathieu and A. Sinclair. Sherali-Adams relaxations of the matching polytope. In 41st annual ACM symposium on Theory of computing, pages 293-302, 2009.

[Rab03] Y. Rabinovich. On average distortion of embedding metrics into the line and into $L_{1}$. In 35th annual ACM symposium on Theory of computing, pages 456-462, 2003.

[RS09] P. Raghavendra and D. Steurer. Integrality gaps for strong SDP relaxations of unique games. In 50th IEEE Symposium on Foundations of Computer Science, pages 575-585, 2009.

[SA90] H. D. Sherali and W. P. Adams. A hierarchy of relaxation between the continuous and convex hull representations. SIAM J. Discret. Math., 3(3):411-430, 1990.

[Sch08] G. Schoenebeck. Linear level Lasserre lower bounds for certain $k$-CSPs. In 49th IEEE Symposium on Foundations of Computer Science, pages 593-602, 2008.

[Shm97] D. Shmoys. Cut problems and their applications to divide-and-conquer. In D. Hochbaum, editor, Approximation Algorithms for NP-Hard Problems. PWS Publishing Company, 1997.

[STT07] G. Schoenebeck, L. Trevisan, and M. Tulsiani. A linear round lower bound for Lovász-Schrijver SDP relaxations of vertex cover. In 22nd Annual IEEE Conference on Computational Complexity, pages 205-216, 2007.

[Tul09] M. Tulsiani. CSP gaps and reductions in the Lasserre hierarchy. In 41st annual ACM symposium on Theory of computing, pages 303-312, 2009.

[Vaz01] V. V. Vazirani. Approximation algorithms. Springer-Verlag, Berlin, 2001.

[WJ04] M. J. Wainwright and M. I. Jordan. Treewidth-based conditions for exactness of the SheraliAdams and Lasserre relaxations. Technical Report 671, University of California, Berkeley, Department of Statistics, September 2004. 


\section{A NP-hardness for pathwidth 2}

Theorem A.1. Sparsest-Cut (with general demands) is NP-hard even on graphs of pathwidth 2.

Proof. The theorem follows from the following reduction from Max-Cut. Let graph $G=(V, E)$ be an instance of Max-Cut. Construct an instance of Sparsest-Cut on the graph $K_{2, n}$ as follows: identify every vertex $v_{i} \in V$ with a corresponding vertex $v_{i}^{\prime}$ in the new graph. For every edge $\left(v_{i}, v_{j}\right) \in E$ add a demand pair $\left(v_{i}^{\prime}, v_{j}^{\prime}\right)$ with unit demand. Add two vertices $s, t$ and edges $\left\{\left(s, v_{i}^{\prime}\right)\right\}_{i}$ and $\left\{\left(t, v_{i}^{\prime}\right)\right\}_{i}$. Finally, make $(s, t)$ a demand pair with demand $n^{3}$. Consider some cut $(S, T)$ in the new graph. If $s \in S$ and $t \in T$ then the number of cut edges is exactly $n$. Thus the sparseness of the cut is exactly $n /(D(S, T))=n /\left(n^{3}+\left|E_{G}(S \backslash\{s\}, T \backslash\{t\})\right|\right)$. Therefore the sparsest cut that separates $s$ from $t$ corresponds exactly to the max cut in $G$. It remains to show that the sparsest cut in the new graph must separate $s$ from $t$. Indeed, if $\left(S^{\prime}, T^{\prime}\right)$ is a cut for which $s, t \in S^{\prime}$, then the sparseness of the cut is

$$
\begin{aligned}
\frac{\left|T^{\prime}\right|}{D\left(S^{\prime}, T^{\prime}\right)}=\frac{\left|T^{\prime}\right|}{\left|E_{G}\left(S^{\prime} \backslash\{s, t\}, T^{\prime}\right)\right|} & \geq \frac{n}{n\left|E_{G}\left(S^{\prime} \backslash\{s, t\}, T^{\prime}\right)\right|} \\
& >\frac{n}{n^{3}+\left|E_{G}\left(S^{\prime} \backslash\{s, t\}, T^{\prime}\right)\right|}=\frac{n}{D\left(S^{\prime} \backslash t, T^{\prime} \cap\{t\}\right)} .
\end{aligned}
$$

